\title{
A MULTI-POINT DEGENERATE INTERPOLATION PROBLEM FOR GENERALIZED SCHUR FUNCTIONS
}

\author{
VLADIMIR BOLOTNIKOV
}

\begin{abstract}
The Nevanlinna-Pick-Carathéodory-Fejér interpolation problem with finitely many interpolation conditions is considered in the class $\mathscr{S}_{\kappa}$ of meromorphic functions $f$ with $\kappa$ poles inside the unit disk $\mathbb{D}$ and with $\|f\|_{L^{\infty}(\mathbb{T})} \leqslant 1$. Necessary and sufficient conditions for the existence and for the uniqueness of a solution are given in terms of the Pick matrix $P$ of the problem explicitly determined from interpolation data. In particular it is shown that the problem admits infinitely many solutions if and only if $\kappa$ is not less than the number of nonpositive eigenvalues of $P$. For $\kappa$ equal to the number of nonpositive eigenvalues of $P$, we describe the solution set of the problem. Also we present necessary and sufficient conditions for the existence of a meromorphic function with a given pole multiplicity satisfying interpolation conditions and having the minimal possible $L^{\infty}$-norm on the unit circle $\mathbb{T}$.
\end{abstract}

Mathematics subject classification (2010): 30E05.

Keywords and phrases: Generalized Schur functions, Nevanlinna-Pick-Carathéodory-Fejér interpolation problem, Pick matrix, minimal norm solutions.

\section{REFERENCES}

[1] V. M. Adamjan, D. Z. Arov And M. G. Krein, Analytic properties of the Schmidt pairs of a Hankel operator and the generalized Schur-Takagi problem, Mat. Sb., 86 (1971), 34-75.

[2] D. Alpay, T. Constantinescu, A. Dijksma And J. Rovnyak, Notes on interpolation in the generalized Schur class. I. Applications of realization theory, in: Interpolation theory, systems theory and related topics, Operator Theory Adv. Appl., OT 134, 67-97, Birkhäuser, Basel, 2002.

[3] J. A. BALL, A non-Euclidean Lax-Beurling theorem with applications to matricial Nevanlinna-Pick interpolation, in: Toeplitz centennial, Operator Theory: Adv. Appl., OT 4, 67-84, Birkhüser, Basel 1982.

[4] J. A. Ball, I. Gohberg, And L. Rodman, Interpolation of rational matrix functions, Operator Theory: Adv. Appl., OT 45, Birkhäuser, Basel 1990.

[5] J. A. BAll, J. W. Helton, A Beurling-Lax theorem for the Lie group $\mathrm{U}(m, n)$ which contains most classical interpolation theory, J. Operator Theory, 9, 1 (1983), 107-142.

[6] V. Bolotnikov, On Carathéodory-Fejér problem for generalized Schur functions, Integral Equations Operator Theory, 50, 1 (2004), 9-41.

[7] V. BolotNikov, On inertia of certain structured Hermitian matrices, to appear in: Numerical Methods for Structured Matrices and Applications. The Georg Heinig memorial volume (D.A. Bini, V. Mehrmann, V. Olshevsky, E. Tyrtsyhnikov, M. van Barel, Eds.), Operator Theory: Adv. Appl., OT 199, Birkhäuser, Basel 2009.

[8] V. BolotNiKov, On a multi-point interpolation problem for generalized Schur functions, Operator Theory: Adv. Appl., OT 195, 81-101, Birkhäuser, Basel 2010.

[9] V. Bolotnikov, Nevanlinna-Pick meromorphic interpolation: the degenerate case and minimal norm solutions, J. Math. Anal. Appl., 353 (2009), 642-651.

[10] V. BOLOTNIKOV AND A. KHEIFETS, On negative inertia of Pick matrices associated with generalized Schur functions, Integral Equations Operator Theory, 56, 3 (2006), 323-355.

[11] V. Bolotnikov, A. Kheifets, AND L. Rodman, Jet functions having indefinite CarathéodoryPick matrices, Linear Algebra Appl., 385 (2004), 215-286. 
[12] L. B. Golins KII, A generalization of the matrix Nevanlinna-Pick problem, Izv. Akad. Nauk Armyan. SSR Ser.Mat., 18 (1983), 187-205. (Russian).

[13] I.S. IOHVIDOV, Hankel and Toeplitz matrices and forms. Algebraic theory, Birkhäuser, Boston, Mass., 1982.

[14] M. KALtenB ÄCK AND H. WoRACEK, The Krein formula for generalized resolvents in degenerated inner product spaces, Monatsh. Math., 127, 2 (1999), 119-140.

[15] M. G. KREĬN AND H. LANGER, Über die verallgemeinerten Resolventen und die charakteristische Funktion eines isometrischen Operators im Raume $\Pi_{\kappa}$, Colloq. Math. Soc. János Bolyai, 5 (1972), 353-399.

[16] M. G. KREǏn AND H. LANGER, Über einige Fortsetzungsprobleme, die eng mit der Theorie hermitescher Operatoren im Raume $\Pi_{\kappa}$ zusammenhängen. I. Einige Funktionenklassen und ihre Darstellungen, Math. Nachr., 77 (1977), 187-236.

[17] N. NiKOLSKII, Treatise on the shift operator. Spectral function theory, Grundlehren der Mathematischen Wissenschaften 273, Springer-Verlag, Berlin, 1986.

[18] A. A. Nudelman, A new problem of the type of the moment problem, Dokl. Akad. Nauk SSSR, 233, 5 (1977), 792-795.

[19] T. TAKAGI, On an algebraic problem related to an analytic theorem of Carathéodory and Fejér, Japan J. of Math., 1 (1924), 83-93.

[20] H. WoraceK, Nevanlinna-Pick interpolation: the degenerated case, Linear Algebra Appl., 252 (1997), 141-158. 